\begin{tabular}{|l|l|}
\hline Postprint Version & 1.0 \\
\hline Journal website & http://www.sciencedirect.com/science/journal/07383991 \\
\cline { 2 - 2 } Pubmed link & $\begin{array}{l}\text { http://www.ncbi.nlm.nih.gov/entrez/query.fcgi?db=pubmed\&cmd=Retrieve\&do } \\
\text { pt=Abstract\&list_uids=11013542\&query_hl=14\&itool=pubmed_docsum }\end{array}$ \\
\hline DOI & $10.1016 /$ S0738-3991(99)00107-X
\end{tabular}

* Corresponding author. Tel.: 1 31-30-272-9700; fax: 1 31-30272-9729. E-mail address: j.bensing@nivel.nl (J. Bensing)

Stichting NIVEL, P.O. Box 1568, 3500 BN Utrecht, The Netherlands

0738-3991/00/\$ - see front matter @ 2000 Elsevier Science Ireland Ltd. All rights reserved. PII: S0738-3991(99)00107-X

www.elsevier.com/locate/pateducou

\title{
Editorial
}

\section{Communication: the royal pathway to patient-centered medicine}

\section{Jozien M. Bensing*, Peter F.M. VerhaAK, AleXANdra M. van DUlmen, AdriaAn Ph. Visser}

The papers in this special issue on communication in health care can be summarized in one easy and powerful message: communication is the royal pathway to patient-centered medicine. Approached from different angles, the linkage between communication and patient-centered medicine is the common theme that is covered by all authors. Underlying are two distinct assumptions: the first is, that patient-centered medicine is a relevant aim in health care; the second, that communication is an important tool to achieve that aim. Both assumptions deserve some exploration.

We are at the verge of a new paradigm-shift in health care. Norms and values that were long taken for granted seem to be changing. The authoritative doctor who is supposed to make all medical decisions takes gradually place for a model of shared decision making by physicians and patients together. There also is now a widespread belief that not diseases but patients must be the focus of health care. The most pronounced this paradigm-shift is verbalized by Roter (pp. 5-15) who reactivates in her theoretical contribution George Engels earlier predictions about a major paradigmshift at the turn of the century: "just as the molecular and chemistry oriented sciences were adopted as the 20th century medical paradigm, incorporation of the patient's perspective into medicine's definition of patient need has been suggested as the medical paradigm of the 21st century'. Roter introduces the medical dialogue as 'the fundamental vehicle through which the battle of perspectives is waged and the therapeutical relationship is defined'. The paradigmatic character of patient-centered medicine is also described by Bensing (pp. 17-25) who contrasts it with that other appealing new paradigm, that seems to take health care by storm: evidence-based medicine. While Roter describes the paradigm-battle as a battle between an 'old' and a 'new' paradigm, Bensing's analysis shows the more dangerous perspective of two concurring paradigms that - although both growing in popularity yet seem to belong to different, perhaps even conflicting worlds. In her view communication between physician and patient should and could bridge the gap between these promising new paradigms in health care.

As sympathetic as it is, one could yet wonder what the background is of this widespread enthusiasm for patient-centered medicine. Its implication is a power-shift from physician towards patient, and refraining from power is seldom a voluntary act by the powerful party. Yet it seems to happen, and not only because of the pressure of emancipating patients, but also because many physicians themselves are advocates of this movement. Many physicians sincerely feel that patient-centeredness is a requisite for good quality of care. Arguments are given throughout this Special Issue by various authors. Let us summarize them under three headings. 


\section{PATIENTS AS EXPERTS}

One often mentioned reason is that patients are experts in the experience of their symptoms, and physicians are dependent on patients for this expertise. Physicians can only learn about possible diseases and their impact on the patient by listening to the patients' story. That gives power to the patient. Without a cooperative and informative patient, doctors can not do their job properly. As Salmon and Marchant-Haycox (pp. 105-113) demonstrated in their paper, patients use that power throughout the consultation by steering it in a way that helps them to reach their goals, for instance by withholding crucial information about their symptoms, or - the opposite - by catastrophizing their symptoms and requesting treatment. Salmon and Marchant-Haycox illustrate their point with the case of physical symptoms without a known organic cause. This is an area where the goals of physician and patient can easily diverge, which asks for joint agenda-setting in a negotiating rather than an authorative relationship. This issue of problem-awareness and problem-definition, comes back in a number of papers, for instance with Lelie (pp. 81-89), who shows the relevance of these concepts in the care for chronically ill patients (end stage Renal Disease). We may conclude that the first argument for patient-centered medicine can be found in the central role of patient's self-reported symptomatology in physician's understanding of patient's illness and illness behavior. Since all modern diagnostic technology did not succeed and will probably never succeed in making patients' selfreports redundant, we may also conclude that communication is the evident pathway.

\section{DIFFERENCES BETWEEN PATIENTS IN PREFERENCES}

A second, related argument for the success of patient-centered medicine is the growing insight that patients are different in their preferences in health care, even patients with the same objective diseases and the same symptom patterns. Diversity is more common than uniformity. For a long period health care was delivered from the implicit assumption that all patients were the same (which in fact meant that health care was tuned to middle-class white male). Now we know that this is not the case. In this Issue, Kim et al. (pp. 37-47) show the relevance of a gender-specific and culture-specific approach. In western cultures women tend to be expressive about their health concerns, but in developing countries, it is mostly the men who are active in health communication. The popular approach in western countries to treat couples instead of individuals in family planning could well be a disadvantage for women in developing countries. Other papers show that patients not only vary in gender and ethnicity, (both rather visible characteristics), but also in their (less visible) attitudes towards health care. A convincing example is presented by van den Brink-Muinen et al. (pp. 115-127) who demonstrated in an international comparison study on doctor-patient communication in six different European countries, that even within the western world patients living in countries with different health care systems seem to develop quite distinct patterns of expectations towards health care, which shows up in the medical encounter. Both doctor and patient can have a preference for a more doctor-oriented or a more patient-oriented model. In another empirical study Krupat et al. (pp. 49-59) showed that not only the orientation of physician and patient is important, but also the matching between these two orientations, revealing that patients were highly satisfied when either their physicians had a matching orientation or were more patient-centered than themselves. At a more theoretical level the differentiation in patient preferences is elaborated by both Bensing (pp. 17-25) and Roter (pp. 5-15) who developed slightly different theoretical models for analyzing types of patients, respectively types of doctor-patient relationships. Summarizing the papers in this Issue, we may conclude with regard to our second argument that in health care diversity among patients is the norm and not the exception. We may also safely assume that if this diversity is not (solely) reflected in epidemiological data or demographic characteristics, communication is the pathway to a medicine that is patient-oriented instead of disease oriented.

\section{CHANGING MORBIDITY PATTERNS}

The last argument that could explain the popularity of patient-centered medicine as a new paradigm in health care has to do with changing morbidity patterns in western societies, which leads to changing insights in the respective roles of physician and patient. In most chronic diseases the physician's direct influence on the course of the disease is restricted. Much is dependent on the patient's own adaptation and coping capabilities, which prompts the physician for taking the role of 'teacher' or 'consultant' 
rather than the role of 'expert' or even 'guardian'. This is illustrated by Hargie et al. (pp. 61-70), who showed distinct communication patterns among pharmacists for OTC-encounters as compared to prescription-encounters. The subtlety of this issue is nicely demonstrated by Lelie (pp. 81- 89), who showed that even high-tech medical decisions tend to have strong value components, in which shareddecision making is as relevant as in the more psycho social domains of health care. The third argument refers to the responsibility for health, and more specific for the maintenance of health. It must be clear that if the responsibility shifts towards the patient, the physician's role in health care must be directed to strengthen patient's capabilities to handle this responsibility. Many physicians realize that this is the only way to provide good quality of care. As education and motivation are essential elements in this conception of health care, communication is the pathway to a medicine that is patient-centered rather than doctor-centered.

\section{CONCLUSION}

We believe that, together, these three arguments provide a convincing case for our statement that communication is the royal pathway to patient-centered medicine. But to let this statement rise above the level of a mere slogan two additional requirements have to be met. Firstly: in order to provide an evidence base to this statement, it must be demonstrated beyond doubt, that communication can be studied in sound scientific research. Secondly, in order to produce useful knowledge, it must be demonstrated that communication is not the direct result of personality characteristics of the physician involved, but based on a dynamic set of skills that can be taught and trained. Both issues are addressed in this Special Issue. How communication can be unraveled and operationalized in what Roter (pp. 515) called 'building blocks' is described in several papers (Roter (pp. 5-15); Mead and Bower (pp. 71-80); Hargie et al. (pp. 63-70) and Smith et al. (pp. 27-36). Moreover Mead and Bower (pp. 7180) did an interesting job in comparing different observation instruments which all pretend to measure patient-centered medicine. That communication can be taught (and what elements should be taught) is also described in several papers, for instance by Kruijver et al. (pp. 129-145) who analyze the literature on the evidence base of communication training in nurses, by Smith et al. (pp. 27-36) who present details of an evidence-based training program in health care and by Caris-Verhallen et al. (pp. 91-103) who studied the effects of a video-interaction analysis training among nurses in care for the elderly. As editors of this Special Issue, we are particularly happy with the combination of these two approaches in one issue. For a long period researchers and trainers seemed to belong to different worlds with their own culture, their own conferences, and their own kind of publications. A start of this integration occurred at the conference on "Communication in Health Care, Amsterdam, the Netherlands, 10-12 June 1998 [1]. The articles in this issue are a selection of papers presented at this meeting. In our view, it is this integration of training and teaching within the scientific approach to communication in health care, which turns this particular edition of Patient Education and Counseling into a real Special Issue. And although we realize that still much work has to be done, it is yet with some confidence that at the verge of the new millennium, we chose that challenging name for this Special Issue: Communication: the royal pathway to patient-centered medicine.

\section{REFERENCES}

[1] Verhaak P. Communication in Health Care 1998;34(Suppl. 1):S1-S82. 